\title{
Experimental Study of BaaS, Its Implementation Methods and Advantages and Challenges
}

\author{
Halkar Rachappa \\ Asst. Prof. \& Head Dept. of Computer Science \\ Govt. Commerce \& Management College, \\ BALLARI(Karnataka). \\ email :halkarrg@gmail.com
}

Abstract: - BaaS is the facility to provide backend as a service by various vendors to the companies. The developers will outsource the backend jobs to a private or public vendor which will help them to provide all the backend services using cloud technique at a reasonable cost. The cost will depend upon the types and number of backend services required by the organisation. It can be modified at any point of time and as a result of this the costing will also change. By using the BaaS provided by the vendor, the developers can spend and utilise their time at the front-end coding of applications of the system. In this way efficient software and system can be developed which will have latest techniques and gives efficient performance. The paper will explain the characteristics of backend as a service and how it is beneficial for the organisations. There are certain challenges while shifting the existing backend services to the public cloud services provided by the vendor which will be discussed in this paper.

Keywords: - BaaS, Characteristics of BaaS, Advantages, Disadvantages, Types of BaaS, Steps involved in implementation of BaaS, Tools.

Introduction: -

In the time of distributed computing, companies are looking for vendors that can provide them with number of services in the form of cloud computing. The client needs to pay just for the sort of the administrations being utilized. It is helpful technique for having the administrations at one spot and effectively open. The merchant will see every one of the particulars and prerequisites of the client and afterward execute that multitude of administrations in a single cloud and gives the most ideal bundle for the client to utilize. BaaS is the process of providing backend services to the organisations in the form of local cloud which can be accessed from their organisation. The developers are more interested in having a cloud system where the backend processes or backend tasks can be provided by the vendor. This helps the developers to focus on the front-end features of the project efficiently. There are many advantages of having backend as a service in the form of cloud system. The main advantage is that the developers only need to focus on the front-end features and need not worry about the maintenance and monitoring of the backend tasks which will reduce their time and efforts and can help them to utilise that saved time to perform other crucial tasks. BaaS merchants give pre-composed programming to exercises that occur on servers, like client confirmation, information base administration, remote refreshing, and pop-up messages (for portable applications), as well as distributed storage and facilitating.

Importance of Backend as a Service: -

In order to understand backend as a service it is important to understand the difference between the front end and backend sides of the project or any software application.

$>$ Front End tasks/activities of project: -

- The front-end tasks of any project in an organisation is related to the user interface of the project.

- This means front end of project deals with all the activities that serves as a source of communication between the user and the application.

- The user interface should always be easy to understand so the developers should focus on enhancing the features of the front end of the application so that it can easily be understood and navigated by the user. 
- $\quad$ HTML, Java script, CSS are the languages used to design the front end and are easy and faster to use.

Back-end Tasks/Activities: - [1]

- Back-end services are the activities which involves the implementation of the logic of the application, the coding of the components of the project etc.

- It is responsible for all types of functions, database, how it interacts with server etc.

- It requires highly skilled professionals who knows coding and has the capability to perform efficiently.

$>$ As the tasks involved in front end services are easy and needs knowledge of HTML which is used for designing the user interface, the developers like to outsource all the back-end activities to vendors as these activities are complex and needs high technical skills as well as a lot of time to understand the features of application and then design them.

$>$ Using the concept of cloud computing there are many vendors in the market who provides backend as a service using cloud computing technology.

$>$ The vendors will understand the requirements of the project and then offer a package which will consist of all the necessary back-end services for that particular project and offers them price for those particular services.

$>$ The developers can decide what all services they need and can modify it at any point of time.

$>$ Back end as a service can be provided for mobile based applications as well as web- based applications.

Reasons to choose Backend as a Service: -

$>$ Useful for urgent feedback for the product: -

- There are some applications where the developers need quick feedback for the same.

- In this case the developers can speed up the coding process by outsourcing the back-end activities to the vendor and simultaneously work on the front-end activities for the application.

- In this way the application can be developed in less time and the developers will be able to receive feedback soon.

Useful in case of simple application development: -

- Baas is a quick solution where there are less number of features of the application being developed.

- If the developers decide and knows that there are very less and simple features that need designing then they can use already existing backend services packages.
- As a result of this the time and effort taken to develop the application will be reduced.

$>$ New application development: -

- Backend as a help can be an impermanent answer for permit you to send off your application quicker. Later on, one can move to custom backend to make the application more dependable and secure.

Characteristics/Features of BaaS: - [2]

There is a lot of competition among vendors that provide backend as services. Their goal is to provide latest features which can help the developers to deploy the application in most effective way. Following are the features of BaaS which are useful for the developers: -

1. Multiple level Authentication

2. Social Integration

3. Real Time Database

4. Data Management

5. Email-verification

6. Third party integration

7. File Storage

8. Database optimization

9. Data Back up

10. Data replication and redundancy

1. Multiple Level authentication: -

Multiple level authentication is the safest way of securing the data and the applications.

$>$ Many vendors of BaaS are providing two-level authentication to its clients which makes sure that the applications being developed are safe and all the data and information stored is safe and secure.

2. Social Integration: -

$>\quad$ With regards to picking the best BaaS choice then one should know whether these administrations will permit to let the client to join like Facebook, LinkedIn, Twitter, Apple, and so on.

$>$ All things considered, it is a generally expected practice that the greater part of the objective clients never need to make separate records to login and utilize various applications.

$>$ Along these lines, the coordination of social stages is exceptionally vital to consider. It is on the grounds that these BaaS highlights can assist the clients with signing in or access information with the outsider applications effortlessly.

3. Real time Databases: -

> The BaaS technique helps the real time databases users to store and synchronize data in real time. This feature of Baas helps the users to use multiple devices in real time environment. 
$>$ With the help of real time databases, it becomes easier for the users to use multiple devices and synchronize their data easily and allows them to use the data from web as well as mobile application.

$>$ In case the network is lost, then real time database will use cache to synchronise the data.

4. Data Management: -

$>$ Mobile devices have limited storage and much of the data is stored at third party data providers. Due to this is the mobile is lost or is stolen then the hackers might access the data.

$>$ To avoid such situation, the data is kept safe at a centralised backend system using BaaS technique.

$>$ BaaS provides various methods to encrypt and secure data and also helps the clients to synchronise their data.

5. Email- Verification: -

$>$ With regards to enrolling a client with an email address then it is exceptionally vital to add the component of email check to guarantee better administrations and security in the most ideal manner.

$>$ In this way, email check highlight empowering is significant. Indeed, different BaaS choices are offering email confirmation benefits too to use for the engineers which will allow to utilize this component without rehashing an already solved problem.

6. Third party integration: -

$>$ In many situations, it is required to use data and information from already existing backend system. This is common in mobile applications also where they might require information from third party.

$>$ To help integrate third party information, the BaaS vendors are providing such facilities which will help the user to integrate third party information easily by using API's.

7. File Storage: -

$>$ This is the most important feature of the Baas where the user can store their data and information at centralised cloud without worrying about the storage space.

$>$ They can easily access their data stored (in the form of images, audio, video, files etc) at any time and from anywhere.

$>$ This is not like common storage devices where if the system is hanged then the data is also lost.

$>$ It is centralised cloud system which gives storage space to store the important files and data and information.

8. Database Optimization: -
> Baas vendors also helps the developers by providing solutions and methods to optimize the use of data base so that it can be used efficiently.

$>$ This provides the users to store the data and information related to themselves as well as about the application also.

9. Data Back up: -

$>$ It is very important that the solution should be provided to take back up of the data in case of system failure or system damage etc.

$>$ BaaS is a technique which by default is providing the facility to take back up of the data automatically.

> This way the developers are more attracted and interested to outsource back-end tasks to the BaaS vendors as they need not worry about the monitoring and taking back up of the data as it will automatically be done by the cloud BaaS.

10. Data replication and redundancy: -

$>$ Unwavering quality and server uptime are the main things which each application expected to be fruitful.

Hence, application and data set redundancies are expected to boost uptime.

An ordinary BaaS framework typically covers including application occurrences, load balancer and a set imitation with two information base examples. Different BaaS likewise give adaptable and completely repetitive engineering as a piece of default executions.

Advantages of BaaS: - [3]
Fast

Development
Less Backend engineers front end

Figure 1 Benefits of BaaS as a service.

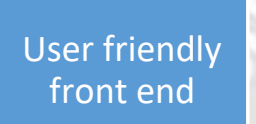

Improved

business
Figure 1

1. Fast Development: - With the help of BaaS, developers find it easy to develop and deploy the application faster. They will outsource the back-end activities like designing and coding of the logical flow of the application to a BaaS vendor so a lot of time is saved and they can simultaneously work on designing user interface as a result of which the applications are developed at a faster speed.

2. Reduced Time for marketing of the product: With the help of Baas, the applications are designed at much faster speed due to which the 
promotion of the applications can be done at much earlier stage. The use of concept of BaaS makes it easy to develop and promote the applications easily and faster than the traditional way of developing the applications.

3. Less backend engineers: - Since the backend services are outsourced to BaaS vendor, now the organisations will not need backend engineers which in turn saves a lot of money.

4. User friendly front end: - Since the majority of the backend services are outsourced, the main focus of the developers will be on the designing of the user interface.

5. Improved performance of the business: - Since a lot of time is saved the business can now focus on ways to improve the business, can spend time in motivating their employees, and look out for ways to improve their other units of the business.

Disadvantages of BaaS: -

Can't be executed completely: - It is absurd to expect to change over all the foundation needs of the association in to BaaS cloud. For this situation the business need to either execute those stages in his own organization or may have to forget about it and quest for different substitutes.

$>$ Terrible showing: - As the administrations given by BaaS is accessible in a cloud, in the event that any of this is down, the representatives will most likely be unable to work and have to stand by till the time the cloud is fully operational.

$>$ Reliance on Vendor: - Organizations getting to the merchant administrations are totally dependent on the sellers for every single necessity. They can't work till the time every one of the administrations are executed by the seller.

$>$ Similarity issues: - If the foundation and foundation of the association isn't carried out in synchronization of the administrations and applications given by the business then because of similarity issues, the presentation of the business will corrupt.

Conclusion: -BaaS is the office to give backend as an assistance by different sellers to the organizations. The designers will rethink the backend occupations to a private or public seller which will assist them with giving all the backend administrations utilizing cloud procedure at a sensible expense. The expense will rely on the kinds and number of backend administrations expected by the association It may be altered anytime of time and because of this the costing will likewise change. By utilizing the BaaS given by the merchant, the engineers can invest and use their energy at the front-end coding of uses of the framework. In this manner productive programming and framework can be created which will have most recent methods and gives effective execution. Back-end administrations are the exercises which includes the execution of the rationale of the application, the coding of the parts of the undertaking and so on. It is liable for a wide range of capacities, information base, how it interfaces with server and so on. It requires exceptionally talented experts who knows coding and has the capacity to perform productively.

\section{References: -}

1.https://clockwise.software/blog/choice-between-mobilebackend-as-a-service-and-custom-backend/

2.https://blog.back4app.com/features-baas-backend-as-aservice/

3.https://blog.back4app.com/what-are-the-benefits-baasbackend-as-a-service/ 